\section{(2) OPEN ACCESS}

\title{
Acupuncture versus sham acupuncture for simple obesity: a systematic review and meta-analysis
}

\author{
Yu-Mei Zhong, ${ }^{1}$ Xiao-Chao Luo, ${ }_{1}$ Yang Chen, ${ }^{1}$ De-Li Lai, ${ }^{1}{ }^{12}$ Wen-Ting Lu, ${ }^{1}$ \\ Ya-Nan Shang, ${ }^{1}$ Lin-Lin Zhang, ${ }^{1}$ Hai-Yan Zhou (1) ${ }^{1}$
}

\begin{abstract}
- Additional material is published online only. To view, please visit the journal online (http://dx.doi.org/10.1136/ postgradmedj-2019-137221).

${ }^{1}$ Acupuncture and Tuina School, Chengdu University of Traditional Chinese Medicine, Chengdu, China

${ }^{2}$ The Affiliated Hospital, School of Medicine, UESTC, Chengdu Women's and Children's Central Hospital, Chengdu, China
\end{abstract}

\section{Correspondence to}

Dr Hai-Yan Zhou, Acupuncture and Tuina School, Chengdu University of Traditional Chinese Medicine, Chengdu 610075,

China;

zhouhaiyan@cdutcm.edu.cn

Received 16 October 2019 Revised 8 December 2019 Accepted 20 December 2019 Published Online First 3 February 2020

\section{ABSTRACT}

Obesity is a growing chronic health problem worldwide. Studies about acupuncture for obesity treatment are many. But there are some doubts about the effectiveness of acupuncture versus sham acupuncture in treating obesity due to its lack of medical evidence. Therefore, the aim of this study is to assess the efficacy of acupuncture for obesity treatment and provide clinic evidence. Four English databases (PubMed, EMBASE, Web of Science and Cochrane Central Register of Controlled Trials) and four Chinese databases (China National Knowledge Infrastructure, Chinese BioMedical Database, Chinese Scientific Journal Database and Wan-Fang Data) were searched from their receptions to August 2019. Randomized controlled trials (RCTs) using the comparison between acupuncture and sham acupuncture to treat simple obesity were included. The primary outcome of body mass index (BMI) would be used to measure the effect of acupuncture on obesity. According to the trial data extraction form based on the Cochrane Handbook, two reviewers separately extracted the data. Risk of bias of the RCTs was assessed by the Cochrane Risk of Bias Tool. The study included 8 RCTs with 403 patients. When compared with sham acupuncture, acupuncture showed obviously effect in $\mathrm{BMI}$ reduction ( $\mathrm{MD}=1.0 \mathrm{~kg} /$ $\mathrm{m} 2,95 \% \mathrm{Cl}=0.6$ to $1.4, \mathrm{P}<0.001)$. There was also significant reduction in body weight $(\mathrm{MD}=1.85 \mathrm{~kg}$, $95 \% \mathrm{Cl}=0.82$ to $2.88, \mathrm{p}<0.001)$, WC $(\mathrm{MD}=0.97 \mathrm{~cm}$, $95 \% \mathrm{Cl}=0.24$ to $1.71, \mathrm{p}=0.01)$ and body fat mass percentage $(\mathrm{MD}=1.01,95 \% \mathrm{Cl}=0.25$ to $1.77, \mathrm{p}<0.05)$. However, WHR (MD=0.01, $95 \% \mathrm{Cl}=0$ to $0.03, p>0.05$ ) was not statistically and significantly different between the acupuncture and control groups. Adverse effects were reported in 3 studies. The review suggests that acupuncture is an effective therapy for simple obesity rather than a placebo effect. This potential benefit needs to be further evaluated by longer-term and more rigorous RCTs.

\section{INTRODUCTION}

Simple obesity is becoming an epidemic health problem that will increase the occurrence of other diseases. Too much fat accumulation in the body is an important manifestation of people with obesity, resulting in the body mass index (BMI) value exceeding the normal level. Over the past few decades, obesity has been thought to be the result of an imbalance between the intake and expenditure of a high-calorie diet. From an evolutionary perspective, the current widespread obesity is mainly caused by sedentary lifestyle, coupled with a high-calorie diet. ${ }^{1}$ The survey demonstrated that the world's mean BMI increased by $>55 \%$ from 1985 to $2017 .^{2}$ Despite decades of efforts to slow down the progress of the epidemic, 39\% of the world's people are obese or overweight at the present time. ${ }^{2}$ The main problem is that obesity is not limited to a certain period of age or to one group of people, but it is common among people of all ages and ethnic groups, leading to an increase of other diseases such as cardiovascular disease, hypertension, osteoarthritis, type 2 diabetes and dyslipidaemia. ${ }^{3-6}$

The diagnosis of obesity depends on the level of BMI. According to WHO's definition, a BMI over $25 \mathrm{~kg} / \mathrm{m}^{2}$ is taken as overweight and a BMI over $30 \mathrm{~kg} / \mathrm{m}^{2}$ as obese. ${ }^{7}$ In addition, WHO considered the physique of the population in the Asia-Pacific region and agreed to define a BMI exceeding $23 \mathrm{~kg} / \mathrm{m}^{2}$ as overweight and a BMI over $25 \mathrm{~kg} / \mathrm{m}^{2}$ as obese. As obesity is a health problem worldwide, there is an increasingly global awareness of the need for effective strategies to prevent and control obesity. Therapeutic strategies for counteracting excess body weight (BW) include dieting, changes of lifestyle, exercise and antiobesity medications. Numerous people with obesity cannot achieve weight loss just through dieting and increasing physical exercise. Although medications such as orlistat and lorcaserin are effective and popular, there exists various limitations due to security reason. ${ }^{8}$ Surgery is also available but it has strict indications and poor patient compliance, that is why surgical treatment cannot be widely used in clinic. ${ }^{9}$ Obesity is closely related to our health and it is necessary to seek effective preventive measures to reduce the occurrence of related diseases at the initial stage in the future. Acupuncture, as a part of traditional Chinese medicine (TCM), is widely used in clinical practice because it is economical, easy and safe. ${ }^{10-13}$ The occurrence of obesity is related to neuroendocrine disorder. The mechanisms of acupuncture in the treatment of obesity might inhibit hyperorexia, regulate the activity of catecholamine neurotransmitter, the 5-hydroxy-tryptamine and ATPase activity in the lateral hypothalamic area (LHA) and reduce the excitation of LHA. ${ }^{14} 15$

Widespread use of acupuncture for the treatment of obesity has been reported by a large number of studies. Their results indicated that acupuncture might have the potential to treat obesity, ${ }^{16-18}$ but there are other reports demonstrating that 
acupuncture is just a placebo effect in clinic. ${ }^{19} 20$ It is of great significance to objectively analyse those results to evaluate the efficacy of acupuncture in obesity treatment. There are also systematic reviews of acupuncture treatment for obesity, which included acupuncture combined with other methods to treat obesity. The results of these reviews cannot evaluate the effect of acupuncture alone. In order to better analyse the effect of acupuncture on obesity and explore whether acupuncture is just a placebo effect, this study performed acupuncture versus sham acupuncture for simple obesity treatment and excluded acupuncture combined with other methods including diet, exercise and medication. We aim to systematically summarise and evaluate the effect of acupuncture therapy on patients with obesity.

\section{MATERIALS AND METHODS}

The study has been registered in PROSPERO (registration number PROSPERO CRD42019129825), it was based on the guidelines of Preferred Reporting Items for Systematic Reviews and Meta Analyses. ${ }^{21}$

\section{Search strategy}

To appraise the efficacy of acupuncture for the treatment of simple obesity, four English databases (EMBASE, PubMed, Cochrane Central Register of Controlled Trials and Web of Science) and four Chinese databases (China National Knowledge Infrastructure, Chinese Scientific Journal Database, Chinese BioMedical Database and Wan-Fang Data) were searched. The study collected randomised controlled trials (RCTs) published from inception to August 2019 without restriction on form and language. We combined the method of Medical Subject Headings term and free words by applying the following terms from English databases: obesity, fat, overweight, acupuncture, electro acupuncture, laser acupuncture auricular acupuncture and needle. Items searched from Chinese databases were Zhen Ci (means acupuncture in Chinese) and Fei Pang (means obesity in Chinese). We also scanned the relevant published references carefully to identify further publications. When there were questions related to the results of the study or the trial design, corresponding authors would be contacted to confirm the information that we extracted from their studies or to eliminate any ambiguity.

\section{Inclusion criteria}

Articles selected should be about RCTs that compared acupuncture with control group (sham acupuncture, placebo acupuncture or no treatment) to assess the efficacy of acupuncture treatment on obesity. Quasi-randomised, comments, case reports, technical reports, animal studies, self-control studies or non-RCTs were excluded. There was no language restriction on studies selection.

All participants were aged over 18 years and were diagnosed with simple obesity (primary, not secondary to other diseases) irrespective of gender. All appropriate definitions of obesity based on BMI that exceeds the normal level were accepted. Pregnancy, patients with serious medical conditions and secondary obesity such as polycystic drug-induced obesity, ovarian syndrome and anterior hypopituitarism were excluded. There was no restriction on race.

The forms of acupuncture therapy included electroacupuncture (EA), body acupuncture, laser acupuncture, classical acupuncture, auricular acupuncture and auricular acupressure. Types of control interventions included sham acupuncture, placebo acupuncture ((1) use acupuncture to insert into skin without penetrating the exact acupoints; (2) use acupuncture to insert into an area where it is near the exact acupoints) and no treatment.

\section{Exclusion criteria}

The following situations were excluded: (1) the reported data were not sufficient to support the results (eg, less number of participants, the means and the SD); (2) the data were duplicate or unextracted; (3) the full text of the article could not be obtained. Studies that combined acupuncture with other therapies such as diet, moxibustion, exercise, medication or message were excluded. Studies with lifestyle intervention such as diet changes and exercise were also excluded, because the aim of this review was to assess the effects of acupuncture treatment alone on obesity.

\section{Outcome measures}

The primary outcome included BMI reduction. The secondary outcome was the reduction of waist circumference (WC), BW, waist-to-hip ratio (WHR) and body fat mass percentage.

\section{Data extraction}

All articles retrieved were imported into endnoteX8 to remove the duplicate studies. The two authors (Y-NS and W-TL) independently scanned the title and the abstract of every record to exclude irrelevant articles. The full text of the qualified articles was investigated and then the authors selected articles that met the inclusion criteria. Every discrepancy was solved by team discussion or consultation with the third reviewer (YC).

Based on the Cochrane Handbook trial data extraction form, the two investigators (Y-NS and W-TL) separately extracted the following data: (1) general information (first author, the year of publication, country, the journal name and so on); (2) participants (the number of participants, gender, age and so on); (3) interventions (type of acupuncture, duration of treatment, study period, acupoints and so on) and comparison interventions (type of treatment, duration, period and so on); (4) outcomes (BMI, BW, WHR, WC and body fat mass percentage) and adverse reactions of the included studies. When there were disagreements between the two reviewers in the process of data extraction, the third author (YC) was consulted to solve the discrepancy. If the data were incomplete, we would contact the first author or the corresponding author (H-YZ).

\section{Quality assessment}

The Cochrane Handbook V.5.3.0 was used to assess the risk of bias of the included RCTs. ${ }^{22}$ The tool included seven items: generation of a random sequence, allocation concealment, blinding of participants and personnel, blinding of outcome assessment, completeness of outcome data, selective of reports and other biases. For each item, the risk of bias for study was rated according to three categories: low risk of bias, high risk of bias or unclear risk of bias. Two reviewers (Y-NS and W-TL) independently assessed the risk of bias of the studies.

\section{Statistical analysis}

We employed mean difference (MD) with 95\% CI to calculate treatment effects of outcomes. $\mathrm{I}^{2}$ was used to assess the statistical heterogeneity among trials. $\mathrm{I}^{2}>50 \%$ indicates that the evidence was heterogeneous, while $\mathrm{I}^{2}<50 \%$ would be taken as the combined results of no heterogeneity. If $\mathrm{p}>0.1$ and $\mathrm{I}^{2}$ $<50 \%$, the fixed effects model would be applied. A random 


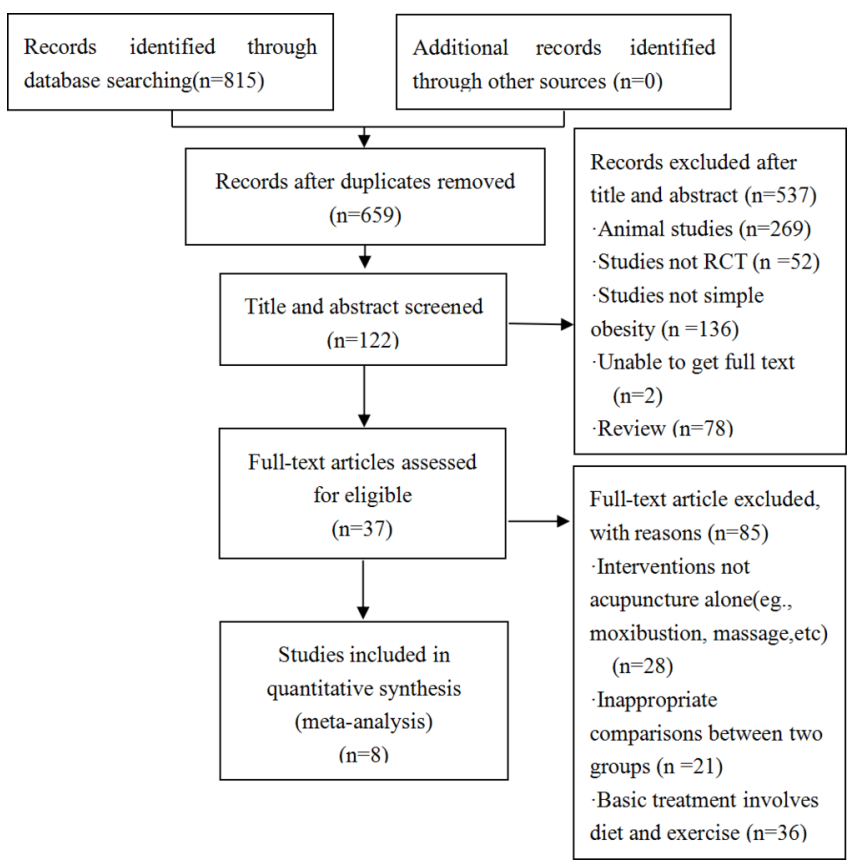

Figure 1 Flow diagram of the number of studies included and excluded. RCT, randomised controlled trial.

effects model would be used when $\mathrm{p}<0.1$ and $\mathrm{I}^{2}>50 \%$. Metaanalysis and statistical calculations were performed by using Cochrane Review Manager (RevMan 5.3) software. $^{23}$

\section{RESULTS}

\section{Literature search and study selection}

Eight hundred fifteen articles were searched through literature retrieval (472 in English and 343 in Chinese) and 156 duplicate articles were removed. We excluded 437 irrelevant articles (including intervention not using acupuncture alone, inappropriate comparison between two groups, basic treatment involves diet and exercise) by scanning the title and abstract. After reading the full text, a total of eight studies meeting the inclusion criteria were obtained. The selection process is summarised in figure 1.

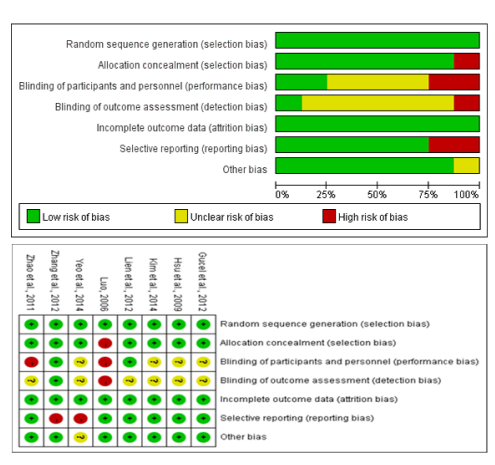

A

B

Figure 2 Risk of bias graph (A) and risk of bias summary (B) of studies included in the meta-analysis.

\section{Study characteristics}

Table 1 presents the characteristics of the eight RCTs which include three studies conducted in China, two in Korea, two in Taiwan and one in Turkey. ${ }^{24-31}$ A total of 403 patients participated in the studies (229 in the treatment groups and 174 in the control group). Among these studies, there were four records ${ }^{25-27} 31$ reporting the effect of auricular acupuncture, three $e^{28-30}$ records reporting the effect of EA and one ${ }^{24}$ record reporting the effect of body acupuncture. The duration of treatment was from 2 to 8 weeks. Three studies reported the adverse events. The study by Zhang et $a l^{29}$ reported a case of bleeding, Lien $e t a l^{27}$ reported a case of dizziness and Hsu et $a l^{25}$ reported one minor inflammation and eight mild tenderness.

\section{Methodological quality}

Figure 2 shows the risk of bias of the included studies. The majority of the included RCTs had more or less methodological weakness according to the quality criteria applied. All records had random allocation. Seven studies ${ }^{24-27} 29-31$ used allocation concealment. Blinding of participants and practitioners was performed only in two included studies ${ }^{27} 29$ due to the particularity of acupuncture therapy. Moreover, one ${ }^{29}$ mentioned the blinding of outcome data and five ${ }^{24-30}$ had a low risk of bias of selective reporting. In addition, all included

Table 1 Characteristics of the included studies

\begin{tabular}{|c|c|c|c|c|c|c|c|}
\hline Literature & Country & $\begin{array}{l}\text { Number of } \\
\text { participants }\end{array}$ & Interventions & Control & $\begin{array}{l}\text { Duration of } \\
\text { treatment } \\
\text { (week) }\end{array}$ & Adverse reactions & Outcomes \\
\hline Güçel et $a l^{24}$ & Turkey & 2020 & Body acupuncture & Sham acupuncture & 5 & Not reported & BMI, BW \\
\hline Hsu et $a l^{25}$ & Taiwan & 2322 & Auricular acupuncture & $\begin{array}{l}\text { Sham auricular } \\
\text { acupuncture }\end{array}$ & 6 & $\begin{array}{l}\text { One minor } \\
\text { inflammation, } \\
\text { eight mild tenderness }\end{array}$ & BMI, BW, WC, \\
\hline Kim et $\left.a\right|^{26}$ & Korea & 2524 & Auricular acupressure & No treatment & 2 & None & $\begin{array}{l}\text { BMI, BW, WHR, body } \\
\text { fat mass } \%\end{array}$ \\
\hline Lien,et $a l^{27}$ & Taiwan & 4823 & Auricular stimulation & $\begin{array}{l}\text { Sham auricular } \\
\text { acupuncture }\end{array}$ & 8 & One dizziness & BMI, BW, WC, WHR \\
\hline $\mathrm{Luo}^{28}$ & China & 2020 & EA & No treatment & 3 & Not reported & BMI, WHR \\
\hline Zhang et $a^{29}$ & China & 1515 & EA & Sham EA & 4 & One bleeding & $\begin{array}{l}\text { BMI, WHR, body fat } \\
\text { mass } \%\end{array}$ \\
\hline Zhao et $\left.a\right|^{30}$ & China & 3535 & EA & Sham EA & 4 & No reported & BMI, BW \\
\hline Yeo et $a \beta^{31}$ & Korea & 4315 & Auricular acupuncture & $\begin{array}{l}\text { Sham auricular } \\
\text { acupuncture }\end{array}$ & 8 & No reported & $\begin{array}{l}\text { BMI, BW, WC, body } \\
\text { fat mass } \%\end{array}$ \\
\hline
\end{tabular}

$B M I$, body mass index; BW, body weight; $E A$, electro-acupuncture; WC, waist circumference ; WHR, waist-to-hip ratio. 


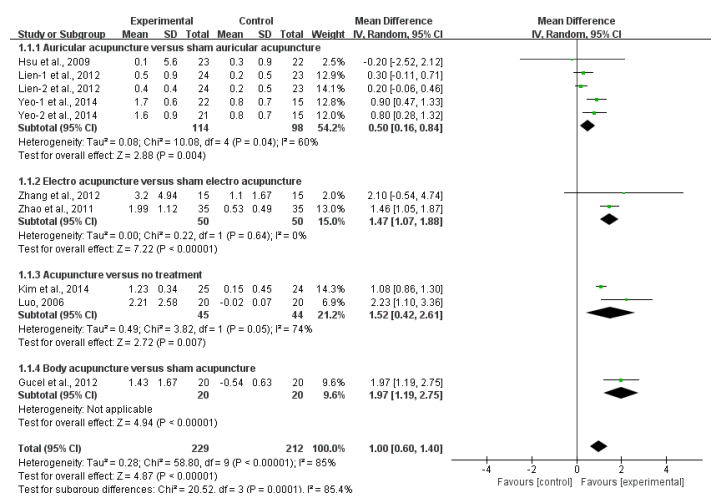

Figure 3 Pooled estimates of body mass index decrements after acupuncture treatment.

RCTs provided complete outcome data and seven ${ }^{24-2931}$ had a low risk of bias in other bias.

\section{Synthesis of results}

\section{Body mass index}

Eight studies reported the reduction in BMI after acupuncture treatment. The high heterogeneity was detected $\left(\mathrm{I}^{2}=85 \%\right.$, $\mathrm{p}<0.1$ ), so the random effects model was used to analysis. The results indicated that there was significant difference in BMI reduction between acupuncture and sham acupuncture $\left(\mathrm{MD}=1.0 \mathrm{~kg} / \mathrm{m}^{2}, 95 \% \mathrm{CI}=0.60\right.$ to $\left.1.40, \mathrm{p}<0.01\right)$. The sensitivity of the eight RCTs was assessed and the results showed that the combined statistical data were still significant and the forest plots had not changed, which indicated that the merged results of meta-analysis were effective.

Subgroup analyses were performed based on different acupuncture therapies. The results showed that auricular acupuncture, EA and body acupuncture significantly reduced BMI compared with control group $\left(\mathrm{MD}=0.50 \mathrm{~kg} / \mathrm{m}^{2}, 95 \% \mathrm{CI}=0.16\right.$ to 0.84 ; $\mathrm{MD}=1.47 \mathrm{~kg} / \mathrm{m}^{2}, 95 \% \quad \mathrm{CI}=1.07$ to $1.88 ; \mathrm{MD}=1.52 \mathrm{~kg} / \mathrm{m}^{2}$, $95 \% \mathrm{CI}=0.42$ to $2.61 ; \mathrm{MD}=1.97 \mathrm{~kg} / \mathrm{m}^{2}, 95 \% \mathrm{CI}=1.19$ to 2.75 ) (figure 3 ). The included studies were $<10$, the publication bias was not carried out.

\section{Body weight}

A total of six records ${ }^{24-273031}$ reported the decrease of BW. Because of the significant heterogeneity among studies $\left(\mathrm{I}^{2}=89.0 \%, \mathrm{p}<0.1\right)$, the random effects model was applied to analyse the BW reduction in obesity $(\mathrm{MD}=1.85 \mathrm{~kg}, 95 \%$ $\mathrm{CI}=0.82$ to $2.88, \mathrm{p}<0.001)$. Subgroup analyses were used to further explore the effects of different acupuncture on BW. Forest plots indicated that auricular acupuncture, EA and body acupuncture reduced BW compared with control group $(\mathrm{MD}=0.98 \mathrm{~kg}, 95 \% \mathrm{CI}=0.10$ to $1.86, \mathrm{p}=0.03 ; \mathrm{MD}=3.78 \mathrm{~kg}$, $95 \% \quad \mathrm{CI}=2.66$ to $4.90, \quad \mathrm{p}<0.001 ; \quad \mathrm{MD}=3.00 \mathrm{~kg}, \quad 95 \%$ $\mathrm{CI}=1.51$ to $4.49, \mathrm{p}<0.001 ; \mathrm{MD}=2.90 \mathrm{~kg}, 95 \% \mathrm{CI}=2.39$ to $3.41, \mathrm{p}<0.001$, respectively) (figure 4).

\section{Waist-to-hip ratio}

Of the eight records, four records ${ }^{26-29}$ showed that acupuncture did not reduce WHR at all compared with control group $(\mathrm{MD}=0.01,95 \% \mathrm{CI}=0$ to $0.03, \mathrm{p}>0.05)$. There was significant heterogeneity among studies $\left(\mathrm{I}^{2}=53 \%, \mathrm{p}<0.1\right)$, so the random effects model was used to analyse the WHR. Subgroup analyses showed that auricular acupuncture, EA, acupuncture did not improve the WHR compared with control group $(\mathrm{MD}=0.01,95 \% \mathrm{CI}=0$ to $0.03, \mathrm{p}>0.05 ; \mathrm{MD}=0.03,95 \%$

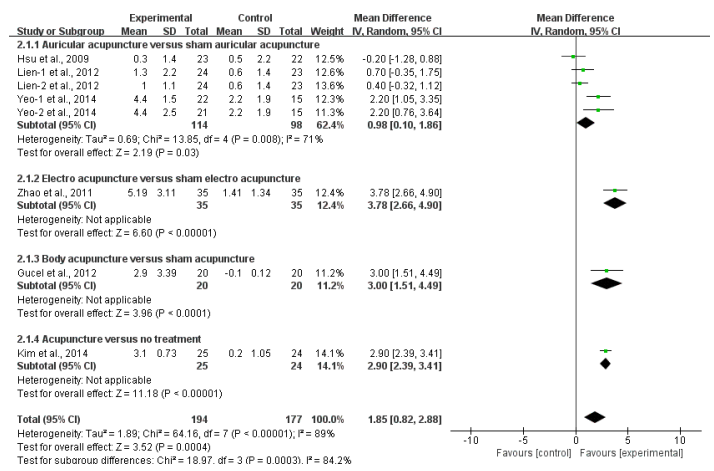

Figure 4 Pooled estimates of body weight decrements after acupuncture treatment.

$\mathrm{CI}=-0.03$ to $0.09, \mathrm{p}>0.05 ; \mathrm{MD}=0.06,95 \% \mathrm{CI}=-0.07$ to $0.19, \mathrm{p}>0.05$ ) (figure 5).

Waist circumference

Three included RCTs ${ }^{25} 2731$ compared changes in WC between auricular acupuncture and sham auricular acupuncture. The results were homogeneous, so the fixed effects model was used $\left(\mathrm{I}^{2}=42 \%, \mathrm{p}>0.1\right)$. Forest plots showed that there were statistically significant differences in WC between the acupuncture treatment and control group $(\mathrm{MD}=0.97 \mathrm{~cm}, 95 \% \mathrm{CI}=0.24$ to $1.71, \mathrm{p}=0.01$ ) (figure 6).

\section{Body fat mass percentage}

Three RCTs ${ }^{26291}$ compared acupuncture treatment with sham acupuncture regarding body fat mass percentage reduction. The results were homogeneous $\left(\mathrm{I}^{2}=0 \%, \mathrm{p}>0.5\right)$ and had a statistical reduction in obesity $(\mathrm{MD}=1.01,95 \% \mathrm{CI}=0.25$ to $1.77, \mathrm{p}<0.05)$. Subgroup analyses showed that auricular acupuncture and acupuncture had no advantage in body fat mass percentage reduction compared with control group $(\mathrm{MD}=0.92,95 \% \mathrm{CI}=-0.19$ to $2.03, \mathrm{p}>0.05 ; \mathrm{MD}=0.78$, $95 \% \mathrm{CI}=-0.40$ to $1.96, \mathrm{p}>0.05)$, whereas EA performed better compared with sham $\mathrm{EA}(\mathrm{MD}=2.20,95 \% \mathrm{CI}=-0.01$ to 4.41, $\mathrm{p}=0.05$ ) (figure 7).

\section{The effect of acupuncture on obesity in different countries and regions}

Subgroup analyses were also used to estimate the effect of acupuncture in different countries or regions. The results indicated that BMI was decremented after acupuncture treatment in China, Korea, Taiwan and Turkey $\left(\mathrm{MD}=1 \mathrm{~kg} / \mathrm{m}^{2}, 95 \%\right.$ $\mathrm{CI}=0.60$ to $1.40, \mathrm{p}<0.001$ ) (figure 8 ). The article also analysed the effect of acupuncture on total cholesterol, triglyceride,

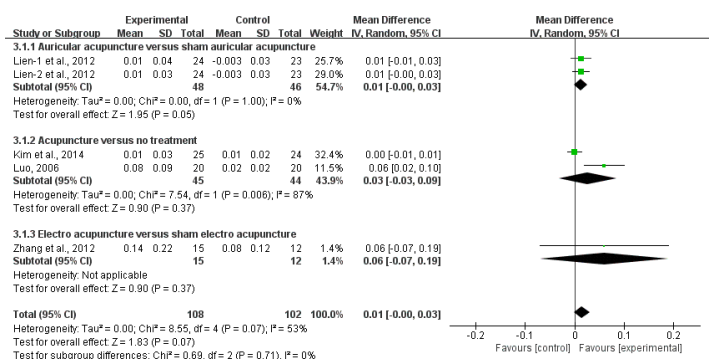

Figure 5 Pooled estimates of waist-to-hip ratio decrements after acupuncture treatment. 


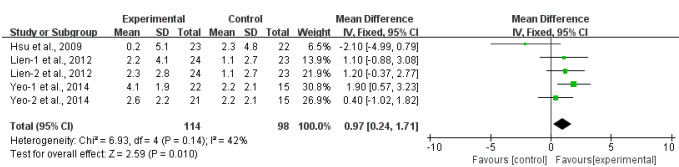

Figure 6 Pooled estimates of waist circumference decrements after acupuncture treatment.

high-density lipoprotein and low-density lipoprotein and the results can be seen in the online supplementary figure 1 .

\section{DISCUSSION}

Obesity is a risk factor for many chronic diseases such as dyslipidaemia, cardiovascular disease and diabetes. ${ }^{32} 33$ It can reduce people's quality of life and damage their physical and mental health. Thus, it is particularly important to stop the occurrence of obesity. There is a large number of studies reported that dieting, exercise, weight loss drug, surgery have efficacy on weight loss. ${ }^{34-36}$ But the treatments mentioned above have more or less limitations, such as the patient's poor persistence of exercise, the uncertain factors referring to the safety of medication and the limited surgery accommodators. As a result, many people are not satisfied with these methods and they tend to use alternative and complementary therapies such as acupuncture to treat obesity.

In recent years, it has been reported that acupuncture is an effective method in improving BMI, BW and WC of patients with obesity. ${ }^{16} 37$ Previous systematic reviews indicated that acupuncture is a safe therapy for patients suffering from hypertension, primary dysmenorrhoea and even patients who take newer oral anticoagulants. ${ }^{37-40}$ But the effectiveness of acupuncture for obesity is controversial due to its lack of medical evidence, and some studies reported that acupuncture may be a placebo effect. ${ }^{414}$ Until now, there is no systematic review of acupuncture (without combining diet or exercise) versus sham acupuncture on the treatment of obesity. In order to further explore whether acupuncture is an effective therapy for obesity, the present study excluded measures including acupuncture combined with dieting, exercise and other treatments compared with the previous meta-analysis. ${ }^{434}$

Here, we selected 8 RCTs including 403 patients suffering from obesity to evaluate the efficacy of acupuncture. Metaanalysis results showed that the reduction level in BMI before and after treatment in the acupuncture group was obviously higher than that of the control group $(p<0.001)$. Subgroup analysis indicated that auricular acupuncture, EA and body acupuncture were effective in reducing BMI of patients with obesity $(p<0.05)$. The forest plot also showed that acupuncture can significantly improve BW, WC and body fat mass percentage compared with sham acupuncture or no treatment $(\mathrm{p}<0.05)$. However, acupuncture had no obvious advantage

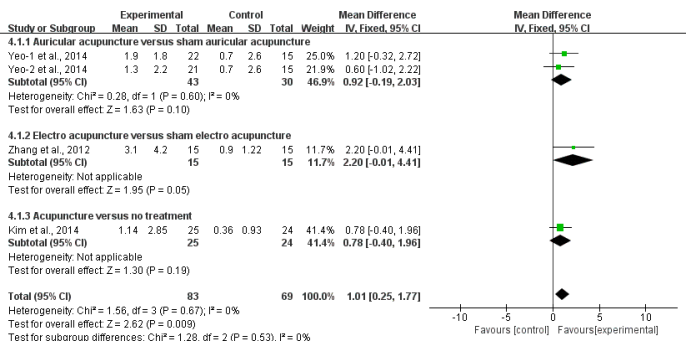

Figure 7 Pooled estimates of body fat mass percentage decrements after acupuncture treatment.

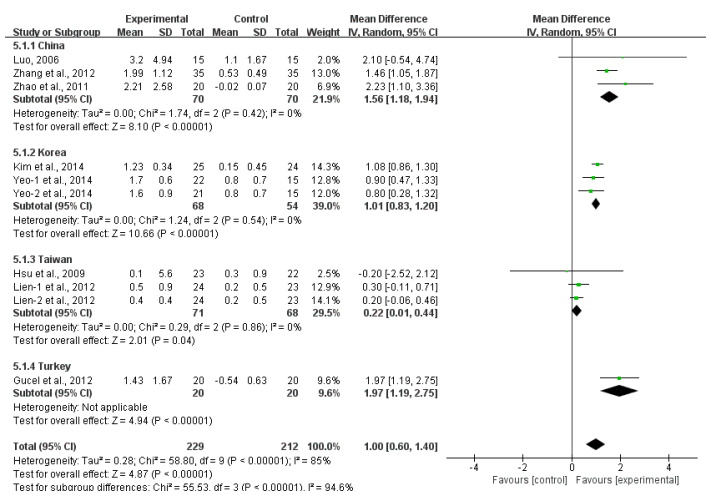

Figure 8 Subgroup analyses of body mass index decrements after acupuncture treatment based on countries or regions.

over sham acupuncture (or no treatment) in improving WHR $(p>0.05)$. Compared with the control group, the improvement of BW after acupuncture treatment was more obvious, which was manifested by the fact that the mean value of weight loss before and after acupuncture in the treatment group was mainly at 3-5, while the figure of the control group was mainly at $0.1-0.5$.

BMI is a more important indicator because it is used to identify obesity and overweight and to analysis the risk of cardiovascular disease, diabetes and other conditions. ${ }^{33}$ The results of our review and analysis are clinically important because they support the possibility that acupuncture contributes to weight loss. What we want to point out is that although acupuncture is used to treat a variety of health problems, its clinical role in obesity management has not been systematically evaluated. The present review reveals that acupuncture is effective in the treatment of obesity, and it is not a placebo effect when comparing with sham acupuncture. Of the eight included studies, four were auricular acupuncture, three were EA and one was body acupuncture. The acupoints of auricular acupuncture therapy are relatively unified, and most of them are shenmen point, stomach point, hunger point and endocrine point. However, the acupoints of EA and body acupuncture are more complicated. In later studies, the selection of acupoints should be unified to reduce clinical heterogeneity.

The forest plot of subgroup analysis was used to explore the effects of auricular acupuncture, EA and body acupuncture in different countries or regions. The results showed that acupuncture significantly reduced BMI compared with sham acupuncture in China, Korea, Taiwan and Turkey. Obesity is a major threat to global health. Acupuncture, as a part of TCM, is more widely used in Asian countries, especially in China, Korea and Japan. There may also be some differences in the effect of acupuncture treatment due to ethnic differences. Clinical studies limited to Asian countries may not be adequately representative. Therefore, the follow-up clinical studies involving more countries and regions should be conducted to better evaluate the therapeutic effects of acupuncture.

The duration of treatment in studies is short, ranging from 2 to 8 weeks. It is worth noting that obesity is a chronic health problem that may require longer periods of acupuncture treatment. However, most studies ignore the long-term effects of acupuncture treatment, which might increase attrition bias. In addition, the weight of people with obesity may fluctuate irrespective of whether they receive treatment or not. So there should be a longer follow-up period, and the results should be 
continuously measured to determine the true effect of acupuncture and its long-term efficacy.

This meta-analysis has some limitations. First, obesity is a global phenomenon, but the studies we included are all from Asian countries or regions, which may not be representative. Second, the different forms of acupuncture (auricular acupuncture, EA and body acupuncture), acupoints and duration of treatment increased the heterogeneity. Third, many of the studies included were of low quality and lacked sufficient levels of blindness. Although blinding of the therapist would be difficult, blinding of participants and outcome assessment should be conducted to minimise the performance and evaluation bias of the trials. Fourth, the number of samples in each trial is small, which might lead to the insufficient sample size for analysis and test efficacy. Therefore, further studies with a larger sample size are required to verify our results.

\section{Main messages}

- Obesity is a growing chronic health problem worldwide.

- The effect of acupuncture on obesity is controversial due to its lack of medical proof and may be a placebo effect.

- This study would be the first study of meta-analysis that compared the acupuncture with sham acupuncture in the treatment of simple obesity.

- The results of this study suggest that acupuncture is effective in the treatment of simple obesity.

\section{Current research questions}

- Whether acupuncture is effective rather than a placebo effect for simple obesity treatment when compared with sham acupuncture?

- Whether acupuncture can be used as a treatment for simple obesity?

- The inadequate implementation of the blind method may be the main deficiency of the methodological quality of the study.

\section{Key references}

1. Lowe $C$, Aiken A, Day AG, et al. Sham acupuncture is as efficacious as true acupuncture for the treatment of IBS: a randomized placebo controlled trial. Neurogastroenterol Motil 2017;29:e13040.

2. Zhang K, Zhou S, Wang C, et al. Acupuncture on obesity: clinical evidence and possible neuroendocrine mechanisms. Evid Based Complement Alternat Med. 2018;2018:6409389.

3. Dos Santos Maciel LY, Dos Santos Leite PM, Neto ML, et al. Comparison of the placebo effect between different non-penetrating acupuncture devices and real acupuncture in healthy subjects: a randomized clinical trial. $B M C$ Complement Altern Med 2016;16:518-29.

4. Fan $\mathrm{XL}, \mathrm{Yu} \mathrm{ML}$, Fu SP, et al. Effectiveness of acupuncture in treatment of simple obesity in animal models: a systematic review and meta-analysis. Evid Based Complement Alternat Med 2019;2019:5459326.

5. Belivani M, Dimitroula C, Katsiki N, et al. Acupuncture in the treatment of obesity: a narrative review of the literature. Acupunct Med 2013;31:88-97.
Self assessment questions

1. What are the types of acupuncture being used to treat obesity?
A. Electro-acupuncture
B. Laser acupuncture
C. Body acupuncture
D. Auricular acupuncture

2. What is the possible mechanism of acupuncture in treating obesity?

A. Inhibit hyperorexia

B. Regulate the activity of catecholamine neurotransmitter

C. Reduce the excitation of lateral hypothalamic area (LHA)

D. Regulate ATPase activity in the LHA

3. What diseases does obesity increase the risk of?
A. Cardiovascular disease
B. Hypertension
C. Dyslipidaemia
D. Type 2 diabetes

4. What percentage of people in the world would be considered obese by 2025 ?
A. $20 \%$
B. $30 \%$
C. $40 \%$
D. $50 \%$

5. What are the main diagnostic criteria for obesity?
A. Body mass index
B. Body weight
C. Waist circumference
D. Waist-to-hip ratio

The results of this meta-analysis demonstrated that acupuncture could improve BMI, BW and WC, suggesting that acupuncture is effective compared with sham acupuncture for the treatment of obesity. However, due to the small number of included studies and limited sample size, our conclusions remain to be confirmed by further high-quality RCTs.

Acknowledgements The authors would like to thank the researchers of the studies included in the meta-analysis.

Contributors $\mathrm{Y}-\mathrm{MZ}, \mathrm{H}-\mathrm{YZ}$ and $\mathrm{D}-\mathrm{LL}$ contributed to the conception and design of the study. Y-MZ drafted the manuscript. W-TL and Y-NS carried out the the data extraction and statistical analysis. $\mathrm{H}-\mathrm{YZ}$ and $\mathrm{YC}$ provided critical insights. $\mathrm{X}-\mathrm{CL}$ and $L-L Z$ reviewed the manuscript. All authors have read and approved the final submitted version of this protocol.

Funding This work was supported by grants from the National Natural Science Foundation of China (No. 81674068 and No. 81973959).

Competing interests None declared.

Patient consent for publication Not required.

Provenance and peer review Not commissioned; externally peer reviewed.

Open access This is an open access article distributed in accordance with the Creative Commons Attribution Non Commercial (CC BY-NC 4.0) license, which permits others to distribute, remix, adapt, build upon this work non-commercially, and license their derivative works on different terms, provided the original work is properly cited, appropriate credit is given, any changes made indicated, and the use is non-commercial. See: http://creativecommons.org/licenses/by-nc/4.0/.

\section{ORCID iD}

Hai-Yan Zhou http://orcid.org/0000-0003-1568-5287

\section{REFERENCES}

1 Lopez KN, Knudson JD. Obesity: from the agricultural revolution to the contemporary pediatric epidemic. Congenit Heart Dis 2012;7:189-99. 
2 World Health Organization. Obesity and overweight, 2015. Available: http://www. who.int/ mediacentre /factsheets/fs311/ en// [Accessed 22 Jan 2017].

3 Tuoyire DA, McNair S, Debrah SA, et al. Perception of risk for hypertension and overweight/obesity in Cape Coast, Ghana. Ghana Med J 2018;52:140-6.

4 Kakoly NS, Earnest A, Teede HJ, et al. The impact of obesity on the incidence of type 2 diabetes among women with polycystic ovary syndrome. Diabetes Care 2019;42:560-7.

5 Yusuf S, Hawken S, Ôunpuu S, et al. Effect of potentially modifiable risk factors associated with myocardial infarction in 52 countries (the INTERHEART study): casecontrol study. The Lancet 2004;364:937-52.

6 Godziuk K, Prado CM, Woodhouse LJ, et al. Prevalence of sarcopenic obesity in adults with end-stage knee osteoarthritis. Osteoarthritis Cartilage 2019;27:1735-45.

7 World Health Organization. Obesity: preventing and managing the global epidemic Report of a WHO consultation. World Health Organ Tech Rep Ser 2000;894:1-253.

8 Khera R, Murad MH, Chandar AK, et al. Association of pharmacological treatments for obesity with weight loss and adverse events: a systematic review and meta-analysis. JAMA 2016:315:2424-34.

9 Slopien R, Horst N, Jaremek JD, et al. The impact of surgical treatment of obesity on the female fertility. Gynecol Endocrinol 2019;35:100-2.

10 Chen JA, Chen JA, Lee S, et al. Potential role for acupuncture in the treatment of food addiction and obesity. Acupunct Med 2018;36:52-5.

11 Liu Z, Yan S, Wu J, et al. Acupuncture for chronic severe functional constipation: a randomized trial. Ann Intern Med 2016;165:761-9.

12 Wu X-K, Stener-Victorin E, Kuang H-Y, et al. Effect of acupuncture and clomiphene in Chinese women with polycystic ovary syndrome: a randomized clinical trial. JAMA 2017;317:2502-14.

13 Hershman DL, Unger JM, Crew K. Acupuncture for aromatase Inhibitor-Related joint pain among breast cancer patients. JAMA 2018;320:2270-1.

14 Liu Z, Sun F, Han Y, et al. [Effect of acupuncture on level of monoamines and activity of adenosine triphosphatase in lateral hypothalamic area of obese rats]. Zhongguo Zhong Xi Yi Jie He Za Zhi 2000;20:521-3.

15 Zhao $\mathrm{M}$, Yuan JH, Li J, et al. Effect of acupuncture on feeding center of hypothalamus in experimental fat rats. Chinese Acupuncture \& Moxibustion 2001;21:49-51.

16 Hung Y-C, Hung I-L, Hu W-L, et al. Reduction in postpartum weight with laser acupuncture: a randomized control trial. Medicine 2016:95:e4716.

17 Darbandi M, Darbandi S, Mobarhan MG, et al. Effects of auricular Acupressure combined with low-calorie diet on the leptin hormone in obese and overweight Iranian individuals. Acupuncture in Medicine 2012;30:208-13.

18 Lei H, Chen X, Liu S, et al. Effect of electroacupuncture on visceral and hepatic fat in women with abdominal obesity: a randomized controlled study based on magnetic resonance imaging. J Altern Complement Med 2017;23:285-94.

19 Lowe C, Aiken A, Day AG, et al. Sham acupuncture is as efficacious as true acupuncture for the treatment of IBS: a randomized placebo controlled trial. Neurogastroenterol Motil 2017;29:e13040.

20 Mazda Y, Kikuchi T, Yoshimatsu A, et al. Acupuncture for reducing pruritus induced by intrathecal morphine at elective cesarean delivery: a placebo-controlled, randomized, double-blind trial. Int J Obstet Anesth 2018;36:66-76.

21 Moher D, Shamseer L, Clarke M, et al. Preferred reporting items for systematic review and meta-analysis protocols (PRISMA-P) 2015 statement. Syst Rev 2015;4:1.

22 Higgins JPT, Altman DG, Gotzsche PC, et al. The Cochrane collaboration's tool for assessing risk of bias in randomised trials. BMJ 2011;343:d5928.

23 Higgins JPT, Green S. Cochrane handbook for systematic reviews of interventions, version 5. 1.0. : The Cochrane Collaboration, 2011.

24 Güçel F, Bahar B, Demirtas C, et al. Influence of acupuncture on leptin, ghrelin, insulin and cholecystokinin in obese women: a randomised, sham-controlled preliminary trial. Acupunct Med 2012;30:203-7.

25 Hsu C-H, Wang C-J, Hwang K-C, et al. The effect of auricular acupuncture in obese women: a randomized controlled trial. J Womens Health 2009;18:813-8.
26 Kim D, Ham OK, Kang C, et al. Effects of auricular acupressure using Sinapsis alba seeds on obesity and self-efficacy in female college students. J Altern Complement Med 2014;20:258-64.

27 Lien C-Y, Liao L-L, Chou P, et al. Effects of auricular stimulation on obese women: a randomized, controlled clinical trial. Eur J Integr Med 2012;4:e45-53.

28 Luo HL. Study on effect and mechanism of elctro-acupuncture on simple obesity. Chongqing Medical University, 2006.

29 Zhang L, Zhou XL, Zhang HM, et al. Clinical effect of electroacupuncture on simple obesity. Journal of Sichuan of Traditional Chinese Medicine 2012;30:134-6.

30 Zhao Y, Liu J, Liu Y, et al. Clinical research of using acupuncture, which can Invigorate spleen and eliminate Phlegm to treat simple obesity. Journal of Sichuan of Traditional Chinese Medicine 2011;29:123-5.

31 Yeo S, Kim KS, Lim S. Randomised clinical trial of five ear acupuncture points for the treatment of overweight people. Acupunct Med 2014;32:132-8.

32 Csige I, Ujvárosy D, Szabó Z, et al. The impact of obesity on the cardiovascular system. J Diabetes Res 2018;2018:1-12.

33 Lee DH, Keum N, Hu FB, et al. Comparison of the association of predicted fat mass, body mass index, and other obesity indicators with type 2 diabetes risk: two large prospective studies in US men and women. Eur J Epidemiol 2018;33:1113-23.

34 Christensen R, Kristensen PK, Bartels EM, et al. Efficacy and safety of the weight-loss drug rimonabant: a meta-analysis of randomized trials. Lancet 2007;370:1706-13.

35 Schafer MJ, Mazula DL, Brown AK, et al. Late-life time-restricted feeding and exercise differentially alter healthspan in obesity. Aging Cell 2019;18:e12966.

36 Arterburn DE, Courcoulas AP. Bariatric surgery for obesity and metabolic conditions in adults. BMJ 2014;349:g3961.

37 Yeh M-L, Chu N-F, Hsu M-YF, et al. Acupoint stimulation on weight reduction for obesity: a randomized sham-controlled study. West I Nurs Res 2015;37:1517-30.

38 Woo HL, Ji HR, Pak YK, et al. The efficacy and safety of acupuncture in women with primary dysmenorrhea: a systematic review and meta-analysis. Medicine 2018:97:e11007.

39 Kwon S, Jung W-S, Yang S, et al. Safety of acupuncture in patients taking newer ora anticoagulants: a retrospective chart review study. Evid Based Complement Alternat Med 2018;2018:1-5.

40 Zhao H, Li D, Li Y, et al. Efficacy and safety of acupuncture for hypertension: an overview of systematic reviews. Complement Ther Clin Pract 2019;34:185-94.

41 dos Santos Maciel LY, dos Santos Leite PM, Neto MLP, et al. Comparison of the placebo effect between different non-penetrating acupuncture devices and real acupuncture in healthy subjects: a randomized clinical trial. BMC Complement Altern Med 2016;16:518-29.

42 Kargozar R, Salari R, Jarahi L, et al. Urtica dioica in comparison with placebo and acupuncture: a new possibility for menopausal hot flashes: a randomized clinical trial. Complement Ther Med 2019;44:166-73.

43 Zhang K, Zhou S, Wang C, et al. Acupuncture on obesity: clinical evidence and possible neuroendocrine mechanisms. Evid Based Complement Alternat Med 2018;2018:1-15.

44 Zhang R-Q, Tan J, Li F-Y, et al. Acupuncture for the treatment of obesity in adults: a systematic review and meta-analysis. Postgrad Med J 2017;93:743-51.

\section{Answer}

1. $(A),(B),(C),(D)$

2. $(A),(B),(C),(D)$

3. $(A),(B),(C),(D)$

4. (C)

5. (A), (B) 